\title{
A Hilton-Milner Theorem for Vector Spaces
}

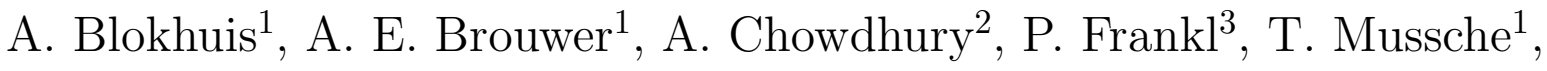 \\ B. Patkós ${ }^{4}$, and T. Szönyi ${ }^{5,6}$ \\ ${ }^{1}$ Dept. of Mathematics, Technological University Eindhoven, \\ P.O. Box 513, 5600 MB Eindhoven, The Netherlands. \\ ${ }^{2}$ Dept. of Mathematics, University of California San Diego, \\ La Jolla, CA 92093, USA. \\ ${ }^{3}$ ShibuYa-Ku, Higashi, 1-10-3-301 Tokyo, 150, Japan. \\ ${ }^{4}$ Department of Computer Science, University of Memphis, \\ TN 38152-3240, USA. \\ ${ }^{5}$ Institute of Mathematics, Eötvös Loránd University, \\ H-1117 Budapest, Pázmány P. s. 1/C, Hungary. \\ ${ }^{6}$ Computer and Automation Research Institute, Hungarian Academy of Sciences, \\ H-1111 Budapest, Lágymányosi ú. 11, Hungary. \\ aartb@win.tue.nl, aeb@cwi.nl, anchowdh@math.ucsd.edu, \\ peter.frankl@gmail.com, bpatkos@memphis.edu, \\ tmussche@gmail.com, szonyi@cs.elte.hu
}

Submitted: Nov 1, 2009; Accepted: May 4, 2010; Published: May 14, 2010 Mathematics Subject Classification: 05D05, 05A30

\begin{abstract}
We show for $k \geqslant 2$ that if $q \geqslant 3$ and $n \geqslant 2 k+1$, or $q=2$ and $n \geqslant 2 k+2$, then any intersecting family $\mathcal{F}$ of $k$-subspaces of an $n$-dimensional vector space over $G F(q)$ with $\bigcap_{F \in \mathcal{F}} F=0$ has size at most $\left[\begin{array}{c}n-1 \\ k-1\end{array}\right]-q^{k(k-1)}\left[\begin{array}{c}n-k-1 \\ k-1\end{array}\right]+q^{k}$. This bound is sharp as is shown by Hilton-Milner type families. As an application of this result, we determine the chromatic number of the corresponding $q$-Kneser graphs.
\end{abstract}

\section{Introduction}

\subsection{Sets}

Let $X$ be an $n$-element set and, for $0 \leqslant k \leqslant n$, let $\left(\begin{array}{l}X \\ k\end{array}\right)$ denote the family of all subsets of $X$ of cardinality $k$. A family $\mathcal{F} \subset\left(\begin{array}{c}X \\ k\end{array}\right)$ is called intersecting if for all $F_{1}, F_{2} \in \mathcal{F}$ we have $F_{1} \cap F_{2} \neq \emptyset$. Erdős, Ko, and Rado [5] determined the maximum size of an intersecting family, and introduced the so-called shifting technique. 
Theorem 1.1 (Erdös-Ko-Rado) Suppose $\mathcal{F} \subset\left(\begin{array}{c}X \\ k\end{array}\right)$ is intersecting and $n \geqslant 2 k$. Then $|\mathcal{F}| \leqslant\left(\begin{array}{c}n-1 \\ k-1\end{array}\right)$. Excepting the case $n=2 k$, equality holds only if $\mathcal{F}=\left\{F \in\left(\begin{array}{l}X \\ k\end{array}\right): x \in F\right\}$ for some $x \in X$.

For any family $\mathcal{F} \subset\left(\begin{array}{l}X \\ k\end{array}\right)$, the covering number $\tau(\mathcal{F})$ is the minimum size of a set that meets all $F \in \mathcal{F}$. Theorem 1.1 shows that if $\mathcal{F} \subset\left(\begin{array}{l}X \\ k\end{array}\right)$ is an intersecting family of maximum size and $n>2 k$, then $\tau(\mathcal{F})=1$.

Hilton and Milner [15] determined the maximum size of an intersecting family with $\tau(\mathcal{F}) \geqslant 2$. Later, Frankl and Füredi [9] gave an elegant proof of Theorem 1.2 using the shifting technique.

Theorem 1.2 (Hilton-Milner) Let $\mathcal{F} \subset\left(\begin{array}{l}X \\ k\end{array}\right)$ be an intersecting family with $k \geqslant 2$, $n \geqslant 2 k+1$, and $\tau(\mathcal{F}) \geqslant 2$. Then $|\mathcal{F}| \leqslant\left(\begin{array}{c}n-1 \\ k-1\end{array}\right)-\left(\begin{array}{c}n-k-1 \\ k-1\end{array}\right)+1$. Equality holds only if

(i) $\mathcal{F}=\{F\} \cup\left\{G \in\left(\begin{array}{l}X \\ k\end{array}\right): x \in G, F \cap G \neq \emptyset\right\}$ for some $k$-subset $F$ and $x \in X \backslash F$.

(ii) $\mathcal{F}=\left\{F \in\left(\begin{array}{l}X \\ 3\end{array}\right):|F \cap S| \geqslant 2\right\}$ for some 3 -subset $S$ if $k=3$.

\section{$1.2 \quad$ Vector spaces}

Theorem 1.1 and Theorem 1.2 have natural extensions to vector spaces. We let $V$ always denote an $n$-dimensional vector space over the finite field $G F(q)$. For $k \in \mathbb{Z}^{+}$, we write $\left[\begin{array}{l}V \\ k\end{array}\right]_{q}$ to denote the family of all $k$-dimensional subspaces of $V$. For $a, k \in \mathbb{Z}^{+}$, define the Gaussian binomial coefficient by

$$
\left[\begin{array}{l}
a \\
k
\end{array}\right]_{q}:=\prod_{0 \leqslant i<k} \frac{q^{a-i}-1}{q^{k-i}-1}
$$

A simple counting argument shows that the size of $\left[\begin{array}{c}6 \\ k\end{array}\right]_{q}$ is $\left[\begin{array}{l}n \\ k\end{array}\right]_{q}$. From now on, we will omit the subscript $q$.

If two subspaces of $V$ intersect in the zero subspace, then we say they are disjoint or that they trivially intersect; otherwise we say the subspaces non-trivially intersect. A family $\mathcal{F} \subset\left[\begin{array}{l}V \\ k\end{array}\right]$ is called intersecting if any two $k$-spaces in $\mathcal{F}$ non-trivially intersect. The maximum size of an intersecting family of $k$-spaces was first determined by Hsieh [16]. For alternate proofs of Theorem 1.3, see [4] and [11]. We remark that there is as yet no analog of the shifting technique for vector spaces.

Theorem 1.3 (Hsieh) Suppose $\mathcal{F} \subset\left[\begin{array}{l}V \\ k\end{array}\right]$ is intersecting and $n \geqslant 2 k$. Then $|\mathcal{F}| \leqslant\left[\begin{array}{c}n-1 \\ k-1\end{array}\right]$. Equality holds if and only if $\mathcal{F}=\left\{F \in\left[\begin{array}{l}V \\ k\end{array}\right]: v \subset F\right\}$ for some one-dimensional subspace $v \subset V$, unless $n=2 k$.

Let the covering number $\tau(\mathcal{F})$ of a family $\mathcal{F} \subset\left[\begin{array}{c}V \\ k\end{array}\right]$ be defined as the minimum dimension of a subspace of $V$ that intersects all elements of $\mathcal{F}$ nontrivially. Theorem 1.3 shows that, as in the set case, if $\mathcal{F}$ is a maximum intersecting family of $k$-spaces, then $\tau(\mathcal{F})=1$. Families satisfying $\tau(\mathcal{F})=1$ are known as point-pencils. 
In this paper, we will extend Theorem 1.2 to vector spaces, and determine the maximum size of an intersecting family $\mathcal{F} \subset\left[\begin{array}{l}V \\ k\end{array}\right]$ with $\tau(\mathcal{F}) \geqslant 2$. For two subspaces $S, T \leqslant V$, we let $S+T \leqslant V$ denote their linear span. We observe that for a fixed 1-subspace $E \leqslant V$ and a $k$-subspace $U$ with $E \nless U$, the family

$$
\mathcal{F}_{E, U}=\{U\} \cup\left\{W \in\left[\begin{array}{l}
V \\
k
\end{array}\right]: E \leqslant W, \operatorname{dim}(W \cap U) \geqslant 1\right\}
$$

is not maximal as we can add all subspaces in $\left[\begin{array}{c}E+U \\ k\end{array}\right]$ that are not in $\mathcal{F}_{E, U}$. We will say that $\mathcal{F}$ is an HM-type family if

$$
\mathcal{F}=\left\{W \in\left[\begin{array}{l}
V \\
k
\end{array}\right]: E \leqslant W, \operatorname{dim}(W \cap U) \geqslant 1\right\} \cup\left[\begin{array}{c}
E+U \\
k
\end{array}\right]
$$

for some $E \in\left[\begin{array}{l}V \\ 1\end{array}\right]$ and $U \in\left[\begin{array}{l}V \\ k\end{array}\right]$ with $E \not Z U$. If $\mathcal{F}$ is an HM-type family, then its size is

$$
|\mathcal{F}|=f(n, k, q):=\left[\begin{array}{l}
n-1 \\
k-1
\end{array}\right]-q^{k(k-1)}\left[\begin{array}{c}
n-k-1 \\
k-1
\end{array}\right]+q^{k} .
$$

The main result of the paper is the following theorem.

Theorem 1.4 Suppose $k \geqslant 3$, and either $q \geqslant 3$ and $n \geqslant 2 k+1$, or $q=2$ and $n \geqslant 2 k+2$. For any intersecting family $\mathcal{F} \subseteq\left[\begin{array}{l}V \\ k\end{array}\right]$ with $\tau(\mathcal{F}) \geqslant 2$, we have $|\mathcal{F}| \leqslant f(n, k, q$ ) (with $f(n, k, q)$ as in (1.1)). Equality holds only if

(i) $\mathcal{F}$ is an HM-type family,

(ii) $\mathcal{F}=\mathcal{F}_{3}=\left\{F \in\left[\begin{array}{l}V \\ k\end{array}\right]: \operatorname{dim}(S \cap F) \geqslant 2\right\}$ for some $S \in\left[\begin{array}{l}V \\ 3\end{array}\right]$ if $k=3$.

Furthermore, if $k \geqslant 4$, then there exists an $\epsilon>0$ (independent of $n, k, q$ ) such that if $|\mathcal{F}| \geqslant(1-\epsilon) f(n, k, q)$, then $\mathcal{F}$ is a subfamily of an HM-type family.

If $k=2$, then a maximal intersecting family $\mathcal{F}$ of $k$-spaces with $\tau(\mathcal{F})>1$ is the family of all 2-subspaces of a 3 -subspace, and the conclusion of the theorem holds.

After proving Theorem 1.4 in Section 2, we apply this result to determine the chromatic number of $q$-Kneser graphs. The vertex set of the $q$-Kneser graph $q K_{n: k}$ is $\left[\begin{array}{l}V \\ k\end{array}\right]$. Two vertices of $q K_{n: k}$ are adjacent if and only if the corresponding $k$-subspaces are disjoint. In [3], the chromatic number of the $q$-Kneser graph $q K_{n: 2}$ is determined, and the minimum colorings are characterized. In [18], the chromatic number of the $q$-Kneser graph is determined in general for $q>q_{k}$. In Section 4, we prove the following theorem.

Theorem 1.5 If $k \geqslant 3$, and either $q \geqslant 3$ and $n \geqslant 2 k+1$, or $q=2$ and $n \geqslant 2 k+2$, then the chromatic number of the $q$-Kneser graph is $\chi\left(q K_{n: k}\right)=\left[\begin{array}{c}n-k+1 \\ 1\end{array}\right]$. Moreover, each color class of a minimum coloring is a point-pencil and the points determining a color are the points of an $(n-k+1)$-dimensional subspace.

In Section 5, we prove the non-uniform version of the Erdős-Ko-Rado theorem.

Theorem 1.6 Let $\mathcal{F}$ be an intersecting family of subspaces of $V$. 
(i) If $n$ is even, then $|\mathcal{F}| \leqslant\left[\begin{array}{c}n-1 \\ n / 2-1\end{array}\right]+\sum_{i>n / 2}\left[\begin{array}{c}n \\ i\end{array}\right]$.

(ii) If $n$ is odd, then $|\mathcal{F}| \leqslant \sum_{i>n / 2}\left[\begin{array}{c}n \\ i\end{array}\right]$.

For even $n$, equality holds only if $\mathcal{F}=\left[\begin{array}{c}V \\ >n / 2\end{array}\right] \cup\left\{F \in\left[\begin{array}{c}V \\ n / 2\end{array}\right]: E \leqslant F\right\}$ for some $E \in\left[\begin{array}{l}V \\ 1\end{array}\right]$, or if $\mathcal{F}=\left[\begin{array}{c}V \\ >n / 2\end{array}\right] \cup\left[\begin{array}{c}U \\ n / 2\end{array}\right]$ for some $U \in\left[\begin{array}{c}V \\ n-1\end{array}\right]$. For odd $n$, equality holds only if $\mathcal{F}=\left[\begin{array}{c}V \\ >n / 2\end{array}\right]$.

Note that Theorem 1.6 follows from the profile polytope of intersecting families which was determined implicitly by Bey [1] and explicitly by Gerbner and Patkós [12], but the proof we present in Section 5 is simple and direct.

\section{Proof of Theorem 1.4}

This section contains the proof of Theorem 1.4 which we divide into two cases.

\subsection{The case $\tau(\mathcal{F})=2$}

For any $A \leqslant V$ and $\mathcal{F} \subseteq\left[\begin{array}{l}V \\ k\end{array}\right]$, let $\mathcal{F}_{A}=\{F \in \mathcal{F}: A \leqslant F\}$. First, let us state some easy technical lemmas.

Lemma 2.1 Let $a \geqslant 0$ and $n \geqslant k \geqslant a+1$ and $q \geqslant 2$. Then

$$
\left[\begin{array}{l}
k \\
1
\end{array}\right]\left[\begin{array}{l}
n-a-1 \\
k-a-1
\end{array}\right]<\frac{1}{(q-1) q^{n-2 k}}\left[\begin{array}{l}
n-a \\
k-a
\end{array}\right] .
$$

Proof. The inequality to be proved simplifies to

$$
\left(q^{k-a}-1\right)\left(q^{k}-1\right) q^{n-2 k}<q^{n-a}-1 .
$$

Lemma 2.2 Let $E \in\left[\begin{array}{l}V \\ 1\end{array}\right]$. If $E \nless L \leqslant V$, where $L$ is an l-subspace, then the number of $k$-subspaces of $V$ containing $E$ and intersecting $L$ is at least $\left[\begin{array}{l}l \\ 1\end{array}\right]\left[\begin{array}{l}n-2 \\ k-2\end{array}\right]-q\left[\begin{array}{l}l \\ 2\end{array}\right]\left[\begin{array}{l}n-3 \\ k-3\end{array}\right]$ (with equality for $l=2$ ), and at most $\left[\begin{array}{l}l \\ 1\end{array}\right]\left[\begin{array}{l}n-2 \\ k-2\end{array}\right]$.

Proof. The $k$-spaces containing $E$ and intersecting $L$ in a 1-dimensional space are counted exactly once in the first term. Those subspaces that intersect $L$ in a 2 -dimensional space are counted $\left[\begin{array}{l}2 \\ 1\end{array}\right]=q+1$ times in the first term and $-q$ times in the second term, thus once overall. If a subspace intersects $L$ in a subspace of dimension $i \geqslant 3$, then it is counted $\left[\begin{array}{l}i \\ 1\end{array}\right]$ times in the first term and $-q\left[\begin{array}{l}i \\ 2\end{array}\right]$ times in the second term, and hence a negative number of times overall.

Our next lemma gives bounds on the size of an HM-type family that are easier to work with than the precise formula mentioned in the introduction.

Lemma 2.3 Let $n \geqslant 2 k+1, k \geqslant 3$ and $q \geqslant 2$. If $\mathcal{F} \subset\left[\begin{array}{l}V \\ k\end{array}\right]$ is an HM-type family, then $\left(1-\frac{1}{q^{3}-q}\right)\left[\begin{array}{l}k \\ 1\end{array}\right]\left[\begin{array}{l}n-2 \\ k-2\end{array}\right]<\left[\begin{array}{l}k \\ 1\end{array}\right]\left[\begin{array}{l}n-2 \\ k-2\end{array}\right]-q\left[\begin{array}{l}k \\ 2\end{array}\right]\left[\begin{array}{l}n-3 \\ k-3\end{array}\right] \leqslant f(n, k, q)=|\mathcal{F}| \leqslant\left[\begin{array}{l}k \\ 1\end{array}\right]\left[\begin{array}{l}n-2 \\ k-2\end{array}\right]$. 
Proof. Since $q\left[\begin{array}{l}k \\ 2\end{array}\right]=\left[\begin{array}{l}k \\ 1\end{array}\right]\left(\left[\begin{array}{l}k \\ 1\end{array}\right]-1\right) /(q+1)$ and $n \geqslant 2 k+1$, the first inequality follows from Lemma 2.1. Let $\mathcal{F}$ be the HM-type family defined by the 1 -space $E$ and the $k$-space $U$. Then $\mathcal{F}$ contains all $k$-subspaces of $V$ containing $E$ and intersecting $U$, so that the second inequality follows from Lemma 2.2. For the last inequality, Lemma 2.2 almost suffices, but we also have to count the $k$-subspaces of $\left[\begin{array}{c}E+U \\ k\end{array}\right]$ that do not contain $E$. Each $(k-1)$-subspace $W$ of $U$ is contained in $q+1$ such subspaces, one of which is $E+W$. On the other hand, $E+W$ was counted at least $q+1$ times since $k \geqslant 3$. This proves the last inequality.

Lemma 2.4 If a subspace $S$ does not intersect each element of $\mathcal{F} \subset\left[\begin{array}{l}V \\ k\end{array}\right]$, then there is a subspace $T>S$ with $\operatorname{dim} T=\operatorname{dim} S+1$ and $\left|\mathcal{F}_{T}\right| \geqslant\left|\mathcal{F}_{S}\right| /\left[\begin{array}{c}k \\ 1\end{array}\right]$.

Proof. There is an $F \in \mathcal{F}$ such that $S \cap F=0$. Average over all $T=S+E$ where $E$ is a 1-subspace of $F$.

Lemma 2.5 If an s-dimensional subspace $S$ does not intersect each element of $\mathcal{F} \subset\left[\begin{array}{l}V \\ k\end{array}\right]$, then $\left|\mathcal{F}_{S}\right| \leqslant\left[\begin{array}{l}k \\ 1\end{array}\right]\left[\begin{array}{c}n-s-1 \\ k-s-1\end{array}\right]$.

Proof. There is an $(s+1)$-space $T$ with $\left[\begin{array}{c}n-s-1 \\ k-s-1\end{array}\right] \geqslant\left|\mathcal{F}_{T}\right| \geqslant\left|\mathcal{F}_{S}\right| /\left[\begin{array}{l}k \\ 1\end{array}\right]$.

Corollary 2.6 Let $\mathcal{F} \subseteq\left[\begin{array}{l}V \\ k\end{array}\right]$ be an intersecting family with $\tau(\mathcal{F}) \geqslant s$. Then for any $i$-space $L \leqslant V$ with $i \leqslant s$ we have $\left|\mathcal{F}_{L}\right| \leqslant\left[\begin{array}{l}k \\ 1\end{array}\right]^{s-i}\left[\begin{array}{c}n-s \\ k-s\end{array}\right]$.

Proof. If $i=s$, then clearly $\left|\mathcal{F}_{L}\right| \leqslant\left[\begin{array}{l}n-s \\ k-s\end{array}\right]$. If $i<s$, then there exists an $F \in \mathcal{F}$ such that $F \cap L=0$; now apply Lemma $2.4 s-i$ times.

Before proving the $q$-analogue of the Hilton-Milner theorem, we describe the essential part of maximal intersecting families $\mathcal{F} \subset\left[\begin{array}{l}V \\ k\end{array}\right]$ with $\tau(\mathcal{F})=2$.

Proposition 2.7 Let $n \geqslant 2 k$ and let $\mathcal{F} \subset\left[\begin{array}{l}V \\ k\end{array}\right]$ be a maximal intersecting family with $\tau(\mathcal{F})=2$. Define $\mathcal{T}$ to be the family of 2-spaces of $V$ that intersect all subspaces in $\mathcal{F}$. One of the following three possibilities holds:

(i) $|\mathcal{T}|=1$ and $\left[\begin{array}{l}n-2 \\ k-2\end{array}\right]<|\mathcal{F}|<\left[\begin{array}{l}n-2 \\ k-2\end{array}\right]+(q+1)\left(\left[\begin{array}{l}k \\ 1\end{array}\right]-1\right)\left[\begin{array}{l}k \\ 1\end{array}\right]\left[\begin{array}{c}n-3 \\ k-3\end{array}\right]$;

(ii) $|\mathcal{T}|>1, \tau(\mathcal{T})=1$, and there is an $(l+1$ )-space $W$ (with $2 \leqslant l \leqslant k$ ) and a 1-space $E \leqslant W$ so that $\mathcal{T}=\{M: E \leqslant M \leqslant W, \operatorname{dim} M=2\}$. In this case,

$\left[\begin{array}{l}l \\ 1\end{array}\right]\left[\begin{array}{l}n-2 \\ k-2\end{array}\right]-q\left[\begin{array}{l}l \\ 2\end{array}\right]\left[\begin{array}{l}n-3 \\ k-3\end{array}\right] \leqslant|\mathcal{F}| \leqslant\left[\begin{array}{l}l \\ 1\end{array}\right]\left[\begin{array}{l}n-2 \\ k-2\end{array}\right]+\left[\begin{array}{l}k \\ 1\end{array}\right]\left(\left[\begin{array}{l}k \\ 1\end{array}\right]-\left[\begin{array}{l}l \\ 1\end{array}\right]\right)\left[\begin{array}{c}n-3 \\ k-3\end{array}\right]+q^{l}\left[\begin{array}{c}n-l \\ k-l\end{array}\right]$.

For $l=2$, the upper bound can be strengthened to

$$
|\mathcal{F}| \leqslant(q+1)\left[\begin{array}{l}
n-2 \\
k-2
\end{array}\right]-q\left[\begin{array}{l}
n-3 \\
k-3
\end{array}\right]+\left[\begin{array}{l}
k \\
1
\end{array}\right]\left(\left[\begin{array}{l}
k \\
1
\end{array}\right]-\left[\begin{array}{l}
2 \\
1
\end{array}\right]\right)\left[\begin{array}{l}
n-3 \\
k-3
\end{array}\right]+q^{2}\left[\begin{array}{l}
k \\
1
\end{array}\right]\left[\begin{array}{l}
n-3 \\
k-3
\end{array}\right]
$$

(iii) $\mathcal{T}=\left[\begin{array}{l}A \\ 2\end{array}\right]$ for some 3-subspace $A$ and $\mathcal{F}=\left\{U \in\left[\begin{array}{l}V \\ k\end{array}\right]: \operatorname{dim}(U \cap A) \geqslant 2\right\}$. In this case, $|\mathcal{F}|=\left(q^{2}+q+1\right)\left(\left[\begin{array}{c}n-2 \\ k-2\end{array}\right]-\left[\begin{array}{c}n-3 \\ k-3\end{array}\right]\right)+\left[\begin{array}{c}n-3 \\ k-3\end{array}\right]$. 
Proof. Let $\mathcal{F} \subset\left[\begin{array}{l}V \\ k\end{array}\right]$ be a maximal intersecting family with $\tau(\mathcal{F})=2$. By maximality, $\mathcal{F}$ contains all $k$-spaces containing a $T \in \mathcal{T}$. Since $n \geqslant 2 k$ and $k \geqslant 2$, two disjoint elements of $\mathcal{T}$ would be contained in disjoint elements of $\mathcal{F}$, which is impossible. Hence, $\mathcal{T}$ is intersecting.

Observe that if $A, B \in \mathcal{T}$ and $A \cap B<C<A+B$, then $C \in \mathcal{T}$. As an intersecting family of 2 -spaces is either a family of 2 -spaces containing some fixed 1-space $E$ or a family of 2 -subspaces of a 3 -space, we get the following:

$(*): \mathcal{T}$ is either a family of all 2 -subspaces containing some fixed 1 -space $E$ that lie in some fixed $(l+1)$-space with $k \geqslant l \geqslant 1$, or $\mathcal{T}$ is the family of all 2 -subspaces of a 3 -space.

(i) : If $|\mathcal{T}|=1$, then let $S$ denote the only 2-space in $\mathcal{T}$ and let $E \leqslant S$ be any 1-space. Since $\tau(\mathcal{F})>1$, there exists an $F \in \mathcal{F}$ with $E \nless F$, for which we must have $\operatorname{dim}(F \cap S)=1$. As $S$ is the only element of $\mathcal{T}$, for any 1 -subspace $E^{\prime}$ of $F$ different from $F \cap S$, we have $\mathcal{F}_{E+E^{\prime}} \leqslant\left[\begin{array}{l}k \\ 1\end{array}\right]\left[\begin{array}{l}n-3 \\ k-3\end{array}\right]$ by Lemma 2.5. Hence the number of subspaces containing $E$ but not containing $S$ is at most $\left(\left[\begin{array}{l}k \\ 1\end{array}\right]-1\right)\left[\begin{array}{l}k \\ 1\end{array}\right]\left[\begin{array}{l}n-3 \\ k-3\end{array}\right]$. This gives the upper bound.

(ii) : Assume that $\tau(\mathcal{T})=1$ and $|\mathcal{T}|>1$. By $(*), \mathcal{T}$ is the set of 2 -spaces in an $(l+1)$ space $W$ (with $l \geqslant 2$ ) containing some fixed 1-space $E$. Every $F \in \mathcal{F} \backslash \mathcal{F}_{E}$ intersects $W$ in a hyperplane. Let $L$ be a hyperplane in $W$ not on $E$. Then $\mathcal{F}$ contains all $k$-spaces on $E$ that intersect $L$. Hence the lower bound and the first term in the upper bound come from Lemma 2.2. The second term comes from using Lemma 2.5 to count the $k$-spaces of $\mathcal{F}$ that contain $E$ and intersect a given $F \in \mathcal{F}$ (not containing $E$ ) in a point of $F \backslash W$. If $l \geqslant 3$, then there are $q^{l}$ hyperplanes in $W$ not containing $E$ and there are $\left[\begin{array}{c}n-l \\ k-l\end{array}\right] k$-spaces through such a hyperplane; this gives the last term. For $l=2$, we use the tight lower bound in Lemma 2.2 to count the number of $k$-spaces on $E$ that intersect $L$. There are $q^{2}$ hyperplanes in $W$, and they cannot be in $\mathcal{T}$, so Lemma 2.5 gives the bound.

(iii) : This is immediate.

Corollary 2.8 Let $\mathcal{F} \subset\left[\begin{array}{l}V \\ k\end{array}\right]$ be a maximal intersecting family with $\tau(\mathcal{F})=2$. Suppose $q \geqslant 3$ and $n \geqslant 2 k+1$, or $q=2$ and $n \geqslant 2 k+2$. If $\mathcal{F}$ is at least as large as an HM-type family and $k>3$, then $\mathcal{F}$ is an HM-type family. If $k=3$, then $\mathcal{F}$ is an HM-type family or an $\mathcal{F}_{3}$-type family.

There exists an $\epsilon>0$ (independent of $n, k, q)$ such that if $k \geqslant 4$ and $|\mathcal{F}|$ is at least $(1-\epsilon)$ times the size of an HM-type family, then $\mathcal{F}$ is an HM-type family.

Proof. Apply Proposition 2.7. Note that the HM-type families are precisely those from case (ii) with $l=k$.

Let $n=2 k+r$ where $r \geqslant 1$. We have $|\mathcal{F}| /\left[\begin{array}{l}n-2 \\ k-2\end{array}\right]<1+\frac{q+1}{(q-1) q^{r}}\left[\begin{array}{l}k \\ 1\end{array}\right]$ in case (i) of Proposition 2.7 by Lemma 2.1. We have $|\mathcal{F}| /\left[\begin{array}{c}n-2 \\ k-2\end{array}\right]<\left(\frac{1}{q}+\frac{1}{(q-1) q^{r}}\right)\left[\begin{array}{l}k \\ 1\end{array}\right]+\frac{q^{2}}{(q-1) q^{r}}$ in case (ii) when $l<k$. In both cases, for $q \geqslant 3$ and $k \geqslant 3$, or $q=2, k \geqslant 4$, and $r \geqslant 2$, this is less than $(1-\epsilon)$ times the lower bound on the size of an HM-type family given in Lemma 2.3. Using the stronger estimate in Lemma 2.3, we find the same conclusion for $q=2, k=3$, and $r \geqslant 2$. 
In case (iii), $\left|\mathcal{F}_{3}\right|=\left[\begin{array}{l}3 \\ 2\end{array}\right]\left[\begin{array}{l}n-2 \\ k-2\end{array}\right]-\frac{q^{3}-q}{q-1}\left[\begin{array}{l}n-3 \\ k-3\end{array}\right]$. For $k \geqslant 4$, this is much smaller than the size of the HM-type families. For $k=3$, the two families have the same size.

Proposition 2.9 Suppose that $k \geqslant 3$ and $n \geqslant 2 k$. Let $\mathcal{F} \subseteq\left[\begin{array}{l}V \\ k\end{array}\right]$ be an intersecting family with $\tau(\mathcal{F}) \geqslant 2$. Let $3 \leqslant l \leqslant k$. If there is an l-space that intersects each $F \in \mathcal{F}$ and

$$
|\mathcal{F}|>\left[\begin{array}{l}
l \\
1
\end{array}\right]\left[\begin{array}{l}
k \\
1
\end{array}\right]^{l-1}\left[\begin{array}{l}
n-l \\
k-l
\end{array}\right],
$$

then there is an $(l-1)$-space that intersects each $F \in \mathcal{F}$.

Proof. By averaging, there is a 1 -space $P$ with $\left|\mathcal{F}_{P}\right| \geqslant|\mathcal{F}| /\left[\begin{array}{l}l \\ 1\end{array}\right]$. If $\tau(\mathcal{F})=l$, then by Corollary 2.6, $|\mathcal{F}| \leqslant\left[\begin{array}{l}l \\ 1\end{array}\right]\left[\begin{array}{l}k \\ 1\end{array}\right]^{l-1}\left[\begin{array}{l}n-l \\ k-l\end{array}\right]$, contradicting the hypothesis.

Corollary 2.10 Suppose $k \geqslant 3$ and either $q \geqslant 3$ and $n \geqslant 2 k+1$, or $q=2$ and $n \geqslant 2 k+2$. Let $\mathcal{F} \subseteq\left[\begin{array}{l}V \\ k\end{array}\right]$ be an intersecting family with $\tau(\mathcal{F}) \geqslant 2$. If $|\mathcal{F}|>\left[\begin{array}{l}3 \\ 1\end{array}\right]\left[\begin{array}{l}k \\ 1\end{array}\right]^{2}\left[\begin{array}{l}n-3 \\ k-3\end{array}\right]$, then $\tau(\mathcal{F})=2$; that is, $\mathcal{F}$ is contained in one of the systems in Proposition 2.7, which satisfy the bound on $|\mathcal{F}|$.

Proof. By Lemma 2.1 and the conditions on $n$ and $q$, the right hand side of (2.2) decreases as $l$ increases, where $3 \leqslant l \leqslant k$. Hence, by Proposition 2.9 , we can find a 2 -space that intersects each $F \in \mathcal{F}$.

Remark 2.11 For $n \geqslant 3 k$, all systems described in Proposition 2.7 occur.

\subsection{The case $\tau(\mathcal{F})>2$}

Suppose that $\mathcal{F} \subset\left[\begin{array}{l}V \\ k\end{array}\right]$ is an intersecting family and $\tau(\mathcal{F})=l>2$. We shall derive a contradiction from $|\mathcal{F}| \geqslant f(n, k, q)$, and even from $|\mathcal{F}| \geqslant(1-\epsilon) f(n, k, q)$ for some $\epsilon>0$ (independent of $n, k, q$ ).

\subsubsection{The case $l=k$}

First consider the case $l=k$. Then $|\mathcal{F}| \leqslant\left[\begin{array}{l}k \\ 1\end{array}\right]^{k}$ by Corollary 2.6. On the other hand,

$$
|\mathcal{F}| \geqslant\left(1-\frac{1}{q^{3}-q}\right)\left[\begin{array}{l}
k \\
1
\end{array}\right]\left[\begin{array}{l}
n-2 \\
k-2
\end{array}\right]>\left(1-\frac{1}{q^{3}-q}\right)\left[\begin{array}{l}
k \\
1
\end{array}\right]^{k-1}\left((q-1) q^{n-2 k}\right)^{k-2}
$$

by Lemma 2.3 and Lemma 2.1. If either $q \geqslant 3, n \geqslant 2 k+1$ or $q=2, n \geqslant 2 k+2$, then either $k=3,(n, k, q)=(9,4,3)$, or $(n, k, q)=(10,4,2)$. If $(n, k, q)=(9,4,3)$ then $f(n, k, q)=$ 3837721 , and $40^{4}=2560000$, which gives a contradiction. If $(n, k, q)=(10,4,2)$, then $f(n, k, q)=153171$, and $15^{4}=50625$, which again gives a contradiction. Hence $k=3$. Now $|\mathcal{F}| \geqslant\left(1-\frac{1}{q^{3}-q}\right)\left[\begin{array}{l}k \\ 1\end{array}\right]\left[\begin{array}{l}n-2 \\ k-2\end{array}\right]$ gives a contradiction for $n \geqslant 8$, so $n=7$. Therefore, if we assume that $n \geqslant 2 k+1$ and either $q \geqslant 3,(n, k) \neq(7,3)$ or $q=2, n \geqslant 2 k+2$ then we are not in the case $l=k$.

It remains to settle the case $n=7, k=l=3$, and $q \geqslant 3$. By Lemma 2.4, we can choose a 1 -space $E$ such that $\left|\mathcal{F}_{E}\right| \geqslant|\mathcal{F}| /\left[\begin{array}{l}3 \\ 1\end{array}\right]$ and a 2 -space $S$ on $E$ such that $\left|\mathcal{F}_{S}\right| \geqslant\left|\mathcal{F}_{E}\right| /\left[\begin{array}{l}3 \\ 1\end{array}\right]$. 
Then $\left|\mathcal{F}_{S}\right|>q+1$ since $|\mathcal{F}|>\left[\begin{array}{l}2 \\ 1\end{array}\right]\left[\begin{array}{l}3 \\ 1\end{array}\right]^{2}$. Pick $F^{\prime} \in \mathcal{F}$ disjoint from $S$ and define $H:=S+F^{\prime}$. All $F \in \mathcal{F}_{S}$ are contained in the 5-space $H$. Since $|\mathcal{F}|>\left[\begin{array}{l}5 \\ 3\end{array}\right]$, there is an $F_{0} \in \mathcal{F}$ not contained in $H$. If $F_{0} \cap S=0$, then each $F \in \mathcal{F}_{S}$ is contained in $S+\left(H \cap F_{0}\right)$; this implies $\left|\mathcal{F}_{S}\right| \leqslant q+1$, which is impossible. Thus, all elements of $\mathcal{F}$ disjoint from $S$ are in $H$.

Now $F_{0}$ must meet $F^{\prime}$ and $S$, so $F_{0}$ meets $H$ in a 2 -space $S_{0}$. Since $\left|\mathcal{F}_{S}\right|>q+1$, we can find two elements $F_{1}, F_{2}$ of $\mathcal{F}_{S}$ with the property that $S_{0}$ is not contained in the 4-space $F_{1}+F_{2}$. Since any $F \in \mathcal{F}$ disjoint from $S$ is contained in $H$ and meets $F_{0}$, it must meet $S_{0}$ and also $F_{1}$ and $F_{2}$. Hence the number of such $F$ 's is at most $q^{5}$. Altogether $|\mathcal{F}| \leqslant q^{5}+\left[\begin{array}{l}2 \\ 1\end{array}\right]\left[\begin{array}{l}3 \\ 1\end{array}\right]^{2}$; the first term comes from counting $F \in \mathcal{F}$ disjoint from $S$ and the second term comes from counting $F \in \mathcal{F}$ on a given one-dimensional subspace $E<S$. This contradicts $|\mathcal{F}| \geqslant\left(1-\frac{1}{q^{3}-q}\right)\left[\begin{array}{l}3 \\ 1\end{array}\right]\left[\begin{array}{l}5 \\ 1\end{array}\right]$.

\subsubsection{The case $l<k$}

Assume, for the moment, that there are two $l$-subspaces in $V$ that non-trivially intersect all $F \in \mathcal{F}$, and that these two $l$-spaces meet in an $m$-space, where $0 \leqslant m \leqslant l-1$. By Corollary 2.6, for each 1-subspace $P$ we have $\left|\mathcal{F}_{P}\right| \leqslant\left[\begin{array}{l}k \\ 1\end{array}\right]^{l-1}\left[\begin{array}{c}n-l \\ k-l\end{array}\right]$, and for each 2-subspace $L$ we have $\left|\mathcal{F}_{L}\right| \leqslant\left[\begin{array}{c}k \\ 1\end{array}\right]^{l-2}\left[\begin{array}{c}n-l \\ k-l\end{array}\right]$. Consequently,

$$
|\mathcal{F}| \leqslant\left[\begin{array}{c}
m \\
1
\end{array}\right]\left[\begin{array}{c}
k \\
1
\end{array}\right]^{l-1}\left[\begin{array}{c}
n-l \\
k-l
\end{array}\right]+\left(\left[\begin{array}{l}
l \\
1
\end{array}\right]-\left[\begin{array}{c}
m \\
1
\end{array}\right]\right)^{2}\left[\begin{array}{c}
k \\
1
\end{array}\right]^{l-2}\left[\begin{array}{c}
n-l \\
k-l
\end{array}\right] .
$$

The upper bound (2.3) is a quadratic in $x=\left[\begin{array}{c}m \\ 1\end{array}\right]$ and is largest at one of the extreme values $x=0$ and $x=\left[\begin{array}{c}l-1 \\ 1\end{array}\right]$. The maximum is taken at $x=0$ only when $\left[\begin{array}{l}l \\ 1\end{array}\right]-\frac{1}{2}\left[\begin{array}{c}k \\ 1\end{array}\right]>\frac{1}{2}\left[\begin{array}{c}l-1 \\ 1\end{array}\right]$; that is, when $k=l$. Since we assume that $l<k$, the upper bound in (2.3) is largest for $m=l-1$. We find

$$
|\mathcal{F}| \leqslant\left[\begin{array}{c}
l-1 \\
1
\end{array}\right]\left[\begin{array}{c}
k \\
1
\end{array}\right]^{l-1}\left[\begin{array}{c}
n-l \\
k-l
\end{array}\right]+\left(\left[\begin{array}{l}
l \\
1
\end{array}\right]-\left[\begin{array}{c}
l-1 \\
1
\end{array}\right]\right)^{2}\left[\begin{array}{l}
k \\
1
\end{array}\right]^{l-2}\left[\begin{array}{c}
n-l \\
k-l
\end{array}\right] .
$$

On the other hand,

$$
|\mathcal{F}| \geqslant\left(1-\frac{1}{q^{3}-q}\right)\left[\begin{array}{l}
k \\
1
\end{array}\right]\left[\begin{array}{c}
n-2 \\
k-2
\end{array}\right]>\left(1-\frac{1}{q^{3}-q}\right)\left[\begin{array}{l}
k \\
1
\end{array}\right]^{l-1}\left[\begin{array}{c}
n-l \\
k-l
\end{array}\right]\left((q-1) q^{n-2 k}\right)^{l-2} .
$$

Comparing these, and using $k>l, n \geqslant 2 k+1$, and $n \geqslant 2 k+2$ if $q=2$, we find either $(n, k, l, q)=(9,4,3,3)$ or $q=2, n=2 k+2, l=3$, and $k \leqslant 5$. If $(n, k, l, q)=(9,4,3,3)$ then $f(n, k, q)=3837721$, while the upper bound is 3508960 , which is a contradiction. If $(n, k, l, q)=(12,5,3,2)$ then $f(n, k, q)=183628563$, while the upper bound is 146766865 , which is a contradiction. If $(n, k, l, q)=(10,4,3,2)$ then $f(n, k, q)=153171$, while the upper bound is 116205 , which is a contradiction. Hence, under our assumption that there are two distinct $l$-spaces that meet all $F \in \mathcal{F}$, the case $2<l<k$ cannot occur.

We now assume that there is a unique $l$-space $T$ that meets all $F \in \mathcal{F}$. We can pick a 1-space $E<T$ such that $\left|\mathcal{F}_{E}\right| \geqslant|\mathcal{F}| /\left[\begin{array}{l}l \\ 1\end{array}\right]$. Now there is some $F^{\prime} \in \mathcal{F}$ not on $E$, so $E$ is in $\left[\begin{array}{l}k \\ 1\end{array}\right]$ lines such that each $F \in \mathcal{F}_{E}$ contains at least one of these lines. Suppose $L$ is one of these lines and $L$ does not lie in $T$; we can enlarge $L$ to an $l$-space that still does not 
meet all elements of $\mathcal{F}$, so $\left|\mathcal{F}_{L}\right| \leqslant\left[\begin{array}{l}k \\ 1\end{array}\right]^{l-1}\left[\begin{array}{l}n-l-1 \\ k-l-1\end{array}\right]$ by Lemma 2.4 and Lemma 2.5. If $L$ does lie on $T$, we have $\left|\mathcal{F}_{L}\right| \leqslant\left[\begin{array}{c}k \\ 1\end{array}\right]^{l-2}\left[\begin{array}{c}n-l \\ k-l\end{array}\right]$ by Corollary 2.6. Hence,

$$
|\mathcal{F}| \leqslant\left[\begin{array}{l}
l \\
1
\end{array}\right]\left|\mathcal{F}_{E}\right| \leqslant\left[\begin{array}{l}
l \\
1
\end{array}\right]\left(\left[\begin{array}{c}
l-1 \\
1
\end{array}\right]\left(\left[\begin{array}{l}
k \\
1
\end{array}\right]^{l-2}\left[\begin{array}{l}
n-l \\
k-l
\end{array}\right]\right)+\left(\left[\begin{array}{c}
k \\
1
\end{array}\right]-\left[\begin{array}{c}
l-1 \\
1
\end{array}\right]\right)\left(\left[\begin{array}{c}
k \\
1
\end{array}\right]^{l-1}\left[\begin{array}{c}
n-l-1 \\
k-l-1
\end{array}\right]\right)\right) .
$$

On the other hand, we have $|\mathcal{F}|>\left(1-\frac{1}{q^{3}-q}\right)\left((q-1) q^{n-2 k}\right)^{l-2}\left[\begin{array}{c}k \\ 1\end{array}\right]^{l-1}\left[\begin{array}{c}n-l \\ k-l\end{array}\right]$. Under our standard assumptions $n \geqslant 2 k+1$ and $n \geqslant 2 k+2$ if $q=2$, this implies $q=2, n=2 k+2$, $l=3$, which gives a contradiction. We showed: If $q \geqslant 3$ and $n \geqslant 2 k+1$ or if $q=2$ and $n \geqslant 2 k+2$, then an intersecting family $\mathcal{F} \subset\left[\begin{array}{l}V \\ k\end{array}\right]$ with $|\mathcal{F}| \geqslant f(n, k, q)$ must satisfy $\tau(\mathcal{F}) \leqslant 2$. Together with Corollary 2.8, this proves Theorem 1.4.

\section{Critical families}

A subspace will be called a hitting subspace (and we shall say that the subspace intersects $\mathcal{F})$, if it intersects each element of $\mathcal{F}$.

The previous results just used the parameter $\tau$, so only the hitting subspaces of smallest dimension were taken into account. A more precise description is possible if we make the intersecting system of subspaces critical.

Definition 3.1 An intersecting family $\mathcal{F}$ of subspaces of $V$ is critical if for any two distinct $F, F^{\prime} \in \mathcal{F}$ we have $F \not \subset F^{\prime}$, and moreover for any hitting subspace $G$ there is a $F \in \mathcal{F}$ with $F \subset G$.

Lemma 3.2 For every non-extendable intersecting family $\mathcal{F}$ of $k$-spaces there exists some critical family $\mathcal{G}$ such that

$$
\mathcal{F}=\left\{F \in\left[\begin{array}{l}
V \\
k
\end{array}\right]: \exists G \in \mathcal{G}, G \subseteq F\right\}
$$

Proof. Extend $\mathcal{F}$ to a maximal intersecting family $\mathcal{H}$ of subspaces of $V$, and take for $\mathcal{G}$ the minimal elements of $\mathcal{H}$.

The following construction and result are an adaptation of the corresponding results from Erdős and Lovász [6]:

Construction 3.3 Let $A_{1}, \ldots, A_{k}$ be subspaces of $V$ such that $\operatorname{dim} A_{i}=i$ and $\operatorname{dim}\left(A_{1}+\right.$ $\left.\cdots+A_{k}\right)=\left(\begin{array}{c}k+1 \\ 2\end{array}\right)$. Define

$$
\mathcal{F}_{i}=\left\{F \in\left[\begin{array}{l}
V \\
k
\end{array}\right]: A_{i} \subseteq F, \operatorname{dim} A_{j} \cap F=1 \text { for } j>i\right\} .
$$

Then $\mathcal{F}=\mathcal{F}_{1} \cup \ldots \cup \mathcal{F}_{k}$ is a critical, non-extendable, intersecting family of $k$-spaces, and $\left|\mathcal{F}_{i}\right|=\left[\begin{array}{c}i+1 \\ 1\end{array}\right]\left[\begin{array}{c}i+2 \\ 1\end{array}\right] \cdots\left[\begin{array}{l}k \\ 1\end{array}\right]$ for $1 \leqslant i \leqslant k$. 
For subsets Erdős and Lovász proved that a critical, non-extendable, intersecting family of $k$-sets cannot have more than $k^{k}$ members. They conjectured that the above construction is best possible but this was disproved by Frankl, Ota and Tokushige [10]. Here we prove the following analogous result.

Theorem 3.4 Let $\mathcal{F}$ be a critical, intersecting family of subspaces of $V$ of dimension at most $k$. Then $|\mathcal{F}| \leqslant\left[\begin{array}{l}k \\ 1\end{array}\right]^{k}$.

Proof. Suppose that $|\mathcal{F}|>\left[\begin{array}{c}k \\ 1\end{array}\right]^{k}$. By induction on $i, 0 \leqslant i \leqslant k$, we find an $i$-dimensional subspace $A_{i}$ of $V$ such that $\left|\mathcal{F}_{A_{i}}\right|>\left[\begin{array}{l}k \\ 1\end{array}\right]^{k-i}$. Indeed, since by induction $\left|\mathcal{F}_{A_{i}}\right|>1$ and $\mathcal{F}$ is critical, the subspace $A_{i}$ is not hitting, and there is an $F \in \mathcal{F}$ disjoint from $A_{i}$. Now all elements of $\mathcal{F}_{A_{i}}$ meet $F$, and we find $A_{i+1}>A_{i}$ with $\left|\mathcal{F}_{A_{i+1}}\right|>\left|\mathcal{F}_{A_{i}}\right| /\left[\begin{array}{l}k \\ 1\end{array}\right]$. For $i=k$ this is a contradiction.

Remark 3.5 For $l \leqslant k$ this argument shows that there are not more than $\left[\begin{array}{l}l \\ 1\end{array}\right]\left[\begin{array}{l}k \\ 1\end{array}\right]^{l-1}$ $l$-spaces in $\mathcal{F}$.

If $l=3$ and $\tau>2$ then for the size of $\mathcal{F}$ the previous remark essentially gives $\left[\begin{array}{l}3 \\ 1\end{array}\right]\left[\begin{array}{l}k \\ 1\end{array}\right]^{2}\left[\begin{array}{c}n-3 \\ k-3\end{array}\right]$, which is the bound in Corollary 2.10.

Modifying the Erdős-Lovász construction (see Frankl [7]), one can get intersecting families with many $l$-spaces in the corresponding critical family.

Construction 3.6 Let $A_{1}, \ldots, A_{l}$ be subspaces with $\operatorname{dim} A_{1}=1$, $\operatorname{dim} A_{i}=k+i-l$ for $i \geqslant 2$. Define $\mathcal{F}_{i}=\left\{F \in\left[\begin{array}{l}V \\ k\end{array}\right]: A_{i} \leqslant F, \operatorname{dim}\left(F \cap A_{j}\right) \geqslant 1\right.$ for $\left.j>i\right\}$. Then $\mathcal{F}_{1} \cup \ldots \cup \mathcal{F}_{l}$ is intersecting and the corresponding critical family has at least $\left[\begin{array}{c}k-l+2 \\ 1\end{array}\right] \ldots\left[\begin{array}{l}k \\ 1\end{array}\right] l$-spaces.

For $n$ large enough the Erdős-Ko-Rado theorem for vector spaces follows from the obvious fact that no critical, intersecting family can contain more than one 1-dimensional member. The Hilton-Milner theorem and the stability of the systems follow from $(*)$ which was used to describe the intersecting systems with $\tau=2$. As remarked above, the fact that the critical family has to contain only spaces of dimension 3 or more limits its size to $O\left(\left[\begin{array}{c}n \\ k-3\end{array}\right]\right)$, if $k$ is fixed and $n$ is large enough. Stronger and more general stability theorems can be found in Frankl [8] for the subset case.

\section{Coloring $q$-Kneser graphs}

In this section, we prove Theorem 1.5. We will need the following result of Bose and Burton [2] and its extension by Metsch [17].

Theorem 4.1 (Bose-Burton) If $\mathcal{E}$ is a family of 1-subspaces of $V$ such that any $k$ subspace of $V$ contains at least one element of $\mathcal{E}$, then $|\mathcal{E}| \geqslant\left[\begin{array}{c}n-k+1 \\ 1\end{array}\right]$. Furthermore, equality holds if and only if $\mathcal{E}=\left[\begin{array}{c}H \\ 1\end{array}\right]$ for some $(n-k+1)$-subspace $H$ of $V$. 
Proposition 4.2 (Metsch) If $\mathcal{E}$ is a family of $\left[\begin{array}{c}n-k+1 \\ 1\end{array}\right]-\varepsilon 1$-subspaces of $V$, then the number of $k$-subspaces of $V$ that are disjoint from all $E \in \mathcal{E}$ is at least $\varepsilon q^{(k-1)(n-k)}$.

Proof of Theorem 1.5. Suppose that we have a coloring with at most $\left[\begin{array}{c}n-k+1 \\ 1\end{array}\right]$ colors. Let $G$ (the good colors) be the set of colors that are point-pencils and let $B$ (the bad colors) be the remaining set of colors. Then $|G|+|B| \leqslant\left[\begin{array}{c}n-k+1 \\ 1\end{array}\right]$. Suppose $|B|=\varepsilon>0$. By Proposition 4.2 , the number of $k$-spaces with a color in $B$ is at least $\varepsilon q^{(k-1)(n-k)}$, so that the average size of a bad color class is at least $q^{(k-1)(n-k)}$. This must be smaller than the size of a HM-type family. Thus, by Lemma 2.3,

$$
q^{(k-1)(n-k)} \leqslant\left[\begin{array}{l}
k \\
1
\end{array}\right]\left[\begin{array}{l}
n-2 \\
k-2
\end{array}\right] .
$$

For $k \geqslant 3$ and $q \geqslant 3, n \geqslant 2 k+1$ or $q=2, n \geqslant 2 k+2$, this is a contradiction. (The weaker form of Proposition 4.2, as stated in [17], suffices unless $q=2, n=2 k+2$.) If $|B|=0$, all color classes are point-pencils, and we are done by Theorem 4.1.

\section{Proof of Theorem 1.6}

Let $a+b=n, a<b$ and let $\mathcal{F}_{a}=\mathcal{F} \cap\left[\begin{array}{l}V \\ a\end{array}\right]$ and $\mathcal{F}_{b}=\mathcal{F} \cap\left[\begin{array}{l}V \\ b\end{array}\right]$. We prove

$$
\left|\mathcal{F}_{a}\right|+\left|\mathcal{F}_{b}\right| \leqslant\left[\begin{array}{l}
n \\
b
\end{array}\right]
$$

with equality only if $\mathcal{F}_{a}=\emptyset$ and $\mathcal{F}_{b}=\left[\begin{array}{l}V \\ b\end{array}\right]$.

Adding up (5.4) for $n / 2<b \leqslant n$ gives the bound on $|\mathcal{F}|$ in Theorem 1.6 if $n$ is odd; adding the result of Greene and Kleitman [14] that states $\left|\mathcal{F}_{n / 2}\right| \leqslant\left[\begin{array}{c}n-1 \\ n / 2-1\end{array}\right]$ proves it for even $n$. For the uniqueness part of Theorem 1.6, we only have to note that if $n$ is even then, by results of Godsil and Newman [13], we must have $\mathcal{F}_{n / 2}=\left\{F \in\left[\begin{array}{c}V \\ { }_{n / 2}\end{array}\right]: E \leqslant F\right\}$ for some $E \in\left[\begin{array}{l}V \\ 1\end{array}\right]$ or $\mathcal{F}_{n / 2}=\left[\begin{array}{c}U \\ n / 2\end{array}\right]$ for some $U \in\left[\begin{array}{c}V \\ n-1\end{array}\right]$.

Now we prove (5.4). Consider the bipartite graph with vertex set $\left(\left[\begin{array}{l}V \\ a\end{array}\right],\left[\begin{array}{l}V \\ b\end{array}\right]\right)$ and join $A \in\left[\begin{array}{l}V \\ a\end{array}\right]$ and $B \in\left[\begin{array}{l}V \\ b\end{array}\right]$ if $A \cap B=0$. Observe that $\mathcal{F}_{a} \cup \mathcal{F}_{b}$ is an independent set in this graph. Now, this graph is regular with degree $q^{a b}$. Therefore any independent set in this graph has size at most $\left[\begin{array}{l}n \\ b\end{array}\right]$ by König's Theorem. Moreover, independent sets of size $\left[\begin{array}{l}n \\ b\end{array}\right]$ can only be $\left[\begin{array}{l}V \\ a\end{array}\right]$ or $\left[\begin{array}{l}V \\ b\end{array}\right]$, but the former is not an intersecting family. This proves (5.4).

\section{Acknowledgements}

Ameera Chowdhury thanks the NSF for supporting her and the Rényi Institute for hosting her while she was an NSF-CESRI fellow during the summer of 2008. Balázs Patkós's research was supported by NSF Grant \#: CCF-0728928. Tamás Szőnyi gratefully acknowledges the financial support of NWO, including the support of the DIAMANT and Spinoza projects. He also thanks the Department of Mathematics at TU/e for the warm hospitality. He was partly supported by OTKA Grants T 49662 and NK 67867. 


\section{References}

[1] C. Bey. Polynomial LYM inequalities. Combinatorica, 25(1):19-38, 2005.

[2] R. C. Bose and R. C. Burton. A characterization of flat spaces in a finite geometry and the uniqueness of the Hamming and the MacDonald codes. J. Combin. Theory, 1:96-104, 1966.

[3] A. Chowdhury, C. Godsil, and G. Royle. Colouring lines in projective space. J. Combin. Theory Ser. A, 113(1):39-52, 2006.

[4] A. Chowdhury and B. Patkós. Shadows and intersections in vector spaces. J. Combin. Theory Ser. A, to appear.

[5] P. Erdős, C. Ko, and R. Rado. Intersection theorems for systems of finite sets. Quart. J. Math. Oxford Ser. (2), 12:313-320, 1961.

[6] P. Erdős and L. Lovász. Problems and results on 3-chromatic hypergraphs and some related questions. In Infinite and finite sets (Colloq., Keszthely, 1973; dedicated to P. Erdős on his 60th birthday), Vol. II, pages 609-627. Colloq. Math. Soc. János Bolyai, Vol. 10. North-Holland, Amsterdam, 1975.

[7] P. Frankl. On families of finite sets no two of which intersect in a singleton. Bull. Austral. Math. Soc., 17(1):125-134, 1977.

[8] P. Frankl. On intersecting families of finite sets. J. Combin. Theory Ser. A, 24(2):146161, 1978.

[9] P. Frankl and Z. Füredi. Nontrivial intersecting families. J. Combin. Theory Ser. A, 41(1):150-153, 1986.

[10] P. Frankl, K. Ota, and N. Tokushige. Covers in uniform intersecting families and a counterexample to a conjecture of Lovász. J. Combin. Theory Ser. A, 74(1):33-42, 1996.

[11] P. Frankl and R. M. Wilson. The Erdős-Ko-Rado theorem for vector spaces. J. Combin. Theory Ser. A, 43(2):228-236, 1986.

[12] D. Gerbner and B. Patkós. Profile vectors in the lattice of subspaces. Discrete Math., 309(9):2861-2869, 2009.

[13] C.D. Godsil and M.W. Newman. Independent sets in association schemes. Combinatorica, 26(4):431-443, 2006.

[14] C. Greene and D.J. Kleitman. Proof techniques in the theory of finite sets. In Studies in combinatorics, MAA Stud. Math. 17, pages 22-79. 1978.

[15] A. J. W. Hilton and E. C. Milner. Some intersection theorems for systems of finite sets. Quart. J. Math. Oxford Ser. (2), 18:369-384, 1967.

[16] W.N. Hsieh. Intersection theorems for systems of finite vector spaces. Discrete Math., 12:1-16, 1975.

[17] K. Metsch. How many $s$-subspaces must miss a point set in $\mathrm{PG}(d, q)$. J. Geom., 86(1-2):154-164, 2006.

[18] T. Mussche. Extremal combinatorics in generalized Kneser graphs. PhD thesis, Technical University Eindhoven, 2009. 